\title{
EXISTENCE OF EXTREMAL METRICS ON COMPACT ALMOST HOMOGENEOUS KÄHLER MANIFOLDS WITH TWO ENDS
}

\author{
DANIEL (ZHUANG-DAN) GUAN
}

\begin{abstract}
In this note we prove the existence and the uniqueness of extremal metrics in every Kähler class of any compact almost homogeneous Kähler manifold with two ends by considering the scalar curvature equations, those manifolds might not be projective. We also prove that there are extremal metrics in some Kähler classes of a completion of the multicanonical line bundle of a Kähler-Einstein manifold of positive Ricci curvature.
\end{abstract}

\section{INTRODUCTION}

It is well known that every Kähler class of a compact Kähler homogeneous space admits a Kähler metric with constant scalar curvature. We may ask the following question:

Can we find any special metric on a compact (not necessarily projective) almost homogeneous space with two ends, in particular, on a completion of a line bundle over a rational homogeneous space?

In $[\mathrm{Cl1}, 2]$ Calabi first started the study of extremal Kähler metrics as a generalization of Kähler-Einstein metrics. He proved that for certain compact complex manifolds there exist extremal metrics, and conjectured that there always exists an extremal metric in a Kähler class. The conjecture would imply that there is a Kähler-Einstein metric on a Fano manifold if and only if the Futaki invariant is zero. Although it was shown in [Lv] and [Bu] that there is no extremal metric on certain compact complex manifolds, those examples are not Fano.

In [Sk] and [KS1, 2] Koiso and Sakane found a method for constructing certain Fano manifolds and proved that there exist Kähler-Einstein metrics on those Fano manifolds if and only if the Futaki invariants are zero. Recently by the same argument Andy Hwang [Hw] proved that there always exist extremal metrics on those Fano manifolds (although we finished our main theorem a little earlier, we expected that he would finish the Fano case at that time). So the existence of Kähler-Einstein metrics follows from Calabi's result. They all

Received by the editors April 18, 1994; originally communicated to the Proceedings of the AMS by Peter Li.

1991 Mathematics Subject Classification. Primary 53C55; Secondary 53C30.

Key words and phrases. Extremal metrics, almost homogeneous manifolds, Kähler-Einstein manifolds, scalar curvature equations. 
considered the symmetric Ricci curvature equation, which is not able to solve the general case.

The Fano case is very restricted. For example, in the dimension 2 case, there are only 3 of them, i.e., $C P^{2}, C P^{1} \times C P^{1}$, and $F_{1}$, the blowing up the $C P^{2}$ at one point; they do not include Calabi's examples. But the general case includes all the Hirzebruch surface $F_{n}$. In this paper, by considering the symmetric scalar curvature equation, a method similar to that of [KS] and [Hw] is employed to simplify the proof of [KS1] and [Hw]. The existence of extremal metrics in a more general case is proved, and the results in [KS1] and [Hw] are obtained as corollaries. In particular, it is proved that every Kähler class of any almost homogeneous compact (not necessarily projective) complex manifold with two ends admits a unique extremal metric up to automorphisms and that every Kähler class of a completion of any multicanonical $C^{*}$ bundle over any compact KählerEinstein manifold with positive first Chern class admits an extremal metric. For example, the examples in [Cl1] are of this type; hence the question raised there by Calabi is solved. The main difference between our method and that of others is that we consider the symmetric scalar curvature equation here instead of the symmetric Ricci curvature equation (see also [Gul]), which enables us to solve the general case. The calculation of the scalar curvature in Lemma 2 and the observation in Lemma 3, as well as the proof of Lemma 7, are essential for our proof.

The same consideration has led to many new results in this direction; similar results for quasi-Einstein metrics have been discussed in [Gu1, 2]. We are examining a scalar curvature flow which is related to the quasi-Einstein metrics; it converges to our quasi-Einstein metrics in many cases [Gu3].

This work was finished in the spring of 1992. Here I express my thanks to the Department of Mathematics of the University of California at Berkeley and Professor S. Kobayashi for support, and to Dr. Andy Hwang for discussing [Hw].

I also thank Professor J. Q. Zhong for leading me into this topic when I was in China. And I thank Professor D'Atri for support, as well as Professor Sakane, for sending me [Sa] and [KS2] when I was at Rutgers.

\section{Preliminaries}

We call a metric $g$ an extremal metric if it is a critical point of the functional $g \rightarrow \int R^{2} \omega^{n}$, where $R$ and $\omega$ are the scalar curvature and the Kähler form of $g$.

The results in this paper can be regarded as a continuation of the papers we mentioned above, and several lemmas from those papers will be stated without proof.

Let $p: L \rightarrow M$ be a holomorphic line bundle over a compact Kähler manifold $M$ and $h$ an Hermitian metric of $L$. Denote by $L^{0}$ the open subset $L \backslash\{0$-section $\}$ of $L$ and let $s \in C^{\infty}\left(L^{0}\right)_{\mathbf{R}}$ be defined by $s(l)=\log |l|_{h}$ $\left(l \in L^{0}\right)$, where ||$_{h}$ is the norm defined by $h$. Now consider a function $t=t(s) \in C^{\infty}\left(L^{0}\right)_{\mathbf{R}}$ which depends only on $s$ and is monotone increasing with respect to $s$ and such that $\min t<0<\max t$.

Given a 1-parameter family of Riemannian metrics $g_{t}$ on $M$, consider a Riemannian metric on $L^{0}$ of the form

$$
\tilde{g}=d t^{2}+(d t \circ \tilde{J})^{2}+g,
$$


where $g(l, m)=p^{*} g_{t(s(l))}(m), m \in M$. A function $u$ depending only on $t$ is defined on $L^{0}$ by $u(t)^{2}=\tilde{g}(H, H)$, where $H$ is the real vector field on $L^{0}$ corresponding to the $\mathbf{R}^{*}$ action on $L^{0}$. Let $U=\int_{0}^{t} u(t) d t$.

Lemma 1 (cf. [KS1]). $\tilde{g}$ is a Kähler if and only if $g_{0}$ is Kähler and $g_{t}=$ $g_{0}-U B$, where $B$ is the curvature of $L$ with respect to $h$.

Throughout this paper, we assume that

(1) $\widehat{L}$ is a compactification of $L^{0}$ and $\tilde{g}$ is the restriction of a Kähler metric of $\widehat{L}$ to $L^{0}$, and

(2) the eigenvalues of $B$ and $r$ with respect to $g_{t}$ are constant on $M$ for each $t$, where $r$ is the Ricci curvature of $g_{0}$.

The main theorem of this paper is

Theorem 1. On $\widehat{L}$ there is an extremal metric in the Kähler class of $\tilde{g}$ if $r$ is nonnegative.

The case in which $r$ has negative eigenvalues may be discussed in a later paper.

Let $\left(z^{1}, \ldots, z^{n}\right)$ be a system of holomorphic local coordinates on $M$, $n=\operatorname{dim}_{\mathrm{C}} M$. Using a trivialization of $L^{0}$, a system of holomorphic local coordinates $\left(z^{0}, \ldots, z^{n}\right)$ on $L^{0}$ is taken so that $\partial / \partial z^{0}=H-\sqrt{-1} \widetilde{J} H$. Let $\hat{\partial}_{i}, \hat{\partial}_{\bar{i}}(0 \leq i \leq n)$ be the partial differentiations $\partial / \partial z^{i}, \partial / \partial \bar{z}^{i}$ on $L^{0}$, and let $\partial_{i}, \partial_{\bar{i}}(1 \leq i \leq n)$ be the partial differentiations $\partial / \partial z^{i}, \partial / \partial \bar{z}^{i}$ on $M$.

Lemma 2 (cf. [KS1]). We have

$$
\tilde{g}_{0 \overline{0}}=2 u^{2}, \quad \tilde{g}_{0 \bar{i}}=2 u \hat{\partial}_{\bar{i}} t, \quad \tilde{g}_{i \bar{j}}=g_{i \bar{j}}+2 \hat{\partial}_{i} t \cdot \hat{\partial}_{\bar{j}} t,
$$

where $1 \leq i, j \leq n$. At the point $P \in L^{0}$ considered, we can choose a local coordinate system around $m=p(P) \in M$ such that $\partial / \partial z^{i} t=0$ at $m$, so $\hat{\partial}_{i} t=\hat{\partial}_{\bar{j}} t=0$ at $P$. If $f$ is a function on $L^{0}$ depending only on $t$, then

$$
\begin{gathered}
\hat{\partial}_{0} \hat{\partial}_{\overline{0}} f=u \frac{d}{d t}\left(u \frac{d f}{d t}\right), \quad \hat{\partial}_{i} \hat{\partial}_{\overline{0}} f=0, \\
\hat{\partial}_{i} \hat{\partial}_{\bar{j}} f=-\frac{1}{2} u B_{i j} \frac{d f}{d t} .
\end{gathered}
$$

The Ricci curvature at this point is

$$
\begin{gathered}
\tilde{r}_{0 \overline{0}}=-u \frac{d}{d t}\left(u \frac{d}{d t} \log \left(u^{2} Q\right)\right), \quad \tilde{r}_{0 \bar{i}}=0, \\
\tilde{r}_{i \bar{j}}=p^{*} r_{0 i \bar{j}}+\frac{1}{2} u \frac{d}{d t} \log \left(u^{2} Q\right) \cdot B_{i \bar{j}},
\end{gathered}
$$

where $Q=\operatorname{det}\left(g_{0}^{-1} \cdot g_{t}\right)$ and $r_{0}=\left(r_{0 i \bar{j}}\right)$ is the Ricci curvature of $g_{0}$. In particular, the scalar curvature is

$$
\widetilde{R}=\frac{\Delta}{Q}-\frac{1}{2 Q} \frac{d}{d U}\left(\frac{d}{d U} Q \varphi\right),
$$

where $\varphi=u^{2}$ as a function of $U$ and $\Delta(U)=Q \sum_{i, j} r_{0 i j} g_{t(U)}^{i \bar{j}}$. We have also $\varphi^{\prime}(\min U)=2, \varphi^{\prime}(\max U)=-2$. 
Proof. Here a proof for the last statement is given. By reducing to the case in which $M$ is a point and $\widehat{L}$ is $C P^{1}$, the statement can be proved by direct calculation. Q.E.D.

Lemma 3 (cf. [FMS], [Mb]). U can be regarded as a moment map corresponding to $(\tilde{g}, \widetilde{J} H)$, and $g_{t}$ the symplectic reduction of $\tilde{g}$ at $U(t)$. Then $\tilde{g}$ is extremal if and only if $\widetilde{R}=a U+b$ for some $a, b \in \mathbf{R}$.

Let $M_{0}=U^{-1}(\min U)$ and $M_{\infty}=U^{-1}(\max U)$; they are complex submanifolds, since they are components of the fixed point set of $H-\sqrt{-1} \widetilde{J} H$ which is semisimple.

Lemma 4. Suppose that there is another Kähler metric $\tilde{g}^{\vee}$ on $\widehat{L}$ in the same Kähler class which is of form (1) on $L^{0}$. Let

$$
g^{\vee}, t^{\vee}, U^{\vee}, Q^{\vee}, \Delta^{\vee}, \varphi^{\vee}, u^{\vee}
$$

be the corresponding metric and the corresponding functions of $s$. Then there is a unique corresponding $t^{\vee}$ such that $g_{0}^{\vee}=g_{0}$. In this case, $\min U^{\vee}=\min U$ (or $\max U^{\vee}=\max U$ ) and $Q^{\vee}=Q, \Delta^{\vee}=\Delta$ hold. So we may write $D=\max U$ and $-d=\min U$. Moreover, let $D_{-d}\left(\right.$ or $\left.D_{D}\right)$ be the codimension of $M_{0}$ (or $\left.M_{\infty}\right)$, if $D_{-d}>1\left(\right.$ or $\left.D_{D}>1\right)$. Then

$$
Q(U)=(1+U / d)^{D_{-d}-1} Q_{-d} \quad\left(\text { or }=(1-U / D)^{D_{D}-1} Q_{D}\right),
$$

where $Q_{-d}\left(\right.$ or $\left.Q_{D}\right)$ is a polynomial such that $Q_{-d}(-d) \neq 0\left(\right.$ or $\left.Q_{D}(D) \neq 0\right)$ and

$$
\begin{gathered}
\Delta(U)=D_{-d}\left(D_{-d}-1\right) \frac{1}{d}\left(1+\frac{U}{d}\right)^{D_{-d}-2} Q_{-d}\left(\bmod \left(1+\frac{U}{d}\right)^{D_{-d}-1}\right) \\
\left(o r=D_{D}\left(D_{D}-1\right) \frac{1}{D}\left(1-\frac{U}{D}\right)^{D_{D}-2} Q_{D}\left(\bmod \left(1-\frac{U}{D}\right)^{D_{D}-1}\right)\right) .
\end{gathered}
$$

Proof. Let $\tilde{g}=\tilde{g}^{\vee}=\hat{\partial} \overline{\hat{\partial}} \phi ;$ then

$$
\tilde{g}_{i \bar{j}}^{\vee}-\tilde{g}_{i \bar{j}}+\frac{1}{2} u \frac{d \phi}{d t} B_{i \bar{j}}=\left(g_{0}\right)_{i \bar{j}}-\left(U-\frac{1}{2} u \frac{d \phi}{d t}\right) B_{i \bar{j}}
$$

for $1 \leq i, j \leq n$, so at $\min U($ or $\max U) \tilde{g}_{i \bar{j}}=\tilde{g}_{i \bar{j}}^{\vee}$; therefore there is a $t_{0}$ such that $g_{t^{\vee}\left(t_{0}\right)}^{\vee}=g_{0}$. By choosing $t^{\vee}$ such that $t^{\vee}\left(t_{0}\right)=0$, one sees that $\min U^{\vee}=\min U, \max U^{\vee}=\max U$, as desired.

The last statement follows from the fact that the scalar curvature $R$ is finite on both $M_{0}$ and $M_{\infty}$. Q.E.D.

\section{THE PROOF OF THE RESULTS}

From Lemma 3 , it can be seen that if $\tilde{g}$ is an extremal metric, then $\widetilde{R}=$ $a U+b$ for some $a, b \in \mathbf{R}$, so by Lemma 2 we have

$$
-\frac{1}{Q} \frac{d}{d U}\left(-2 \int_{-d}^{U} \Delta(x) d x+\frac{d}{d U}(Q \varphi)\right)=2 a U+2 b,
$$


from which we obtain

$$
\frac{d}{d U}(Q \varphi)=-\int_{-d}^{U}(c x+m) Q(x) d x-e+2 \int_{-d}^{U} \Delta(x) d x,
$$

where $c=2 a, m=2 b$. Then

$$
Q \varphi=-\int_{-d}^{U}\left(\int_{-d}^{y}[(c x+m) Q(x)-2 \Delta(x)] d x+e\right) d y .
$$

Denoting the left side by $\Phi(U)$, we have the equations

$$
\begin{gathered}
2 Q(-d)=-e, \\
-2 Q(D)=-\int_{-d}^{D}[(c x+m) Q(x)-2 \Delta(x)] d x-e, \\
0=\int_{-d}^{D}\left(\int_{-d}^{y}[(c x+m) Q(x)-2 \Delta(x)] d x+e\right) d y .
\end{gathered}
$$

Lemma 5. There is a unique solution $c, m, e$ for (2).

Proof. From the first equation in (2), $e$ can be found. Exchanging the order of the integrals gives

$$
\begin{aligned}
\int_{-d}^{U} & \left(\int_{-d}^{y}[(c x+m) Q(x)-2 \Delta(x)] d x\right) d y \\
& =\int_{-d}^{U}[(c x+m) Q(x)-2 \Delta(x)](U-x) d x .
\end{aligned}
$$

The third equation in (2) becomes

$$
\begin{aligned}
\int_{-d}^{D}(c x+m) x Q(x) d x \\
\quad=\int_{-d}^{D}(2 y \Delta(y)+D[(c y+m) Q(y)-2 \Delta(y)]+e) d y .
\end{aligned}
$$

From the second equation in (2), the second term of the right side is independent of $c$ or $m$; i.e., the right side is independent of $c$ or $m$. So the equations (2) become

$$
\beta c+\alpha m=f, \quad \gamma c+\beta m=g
$$

where $\alpha=\int_{-d}^{D} Q(x) d x, \beta=\int_{-d}^{D} x Q(x) d x, \gamma=\int_{-d}^{D} x^{2} Q(x) d x$, and $f$ and $g$ are constants. So there is a unique solution for $c, m$. Q.E.D.

Lemma 6 (cf. [KS1]). There is an extremal metric in the Kähler class of $\tilde{g}$ if $\varphi=\Phi / Q$ is positive on $(-d, D)$.

Lemma 7. $\Phi$ above is always positive on $(-d, D)$ if $r$ is nonnegative.

Proof. First, the derivative of $Q \varphi$ is $2 Q(-d)$ (or $-2 Q(D)$ ) at $-d$ (or $D$ ), which is positive (or negative) in the case $D_{-d}=1$ (or $D_{D}=1$ ). If $D_{-d}>1$ (or $D_{D}>1$ ), then the derivation is positive (or negative) near $-d$ (or $D$ ) by the last statement in Lemma 4.

Second, we have

$$
\frac{d^{2}}{d U^{2}}(Q \varphi)=2 \Delta(U)-(c U+m) Q(U) .
$$


Diagonalizing $B$, we see that $Q$ is a product of polynomials of degree 1 . Let $-a_{1}^{-1}<\cdots<-a_{q}^{-1}<b_{1}^{-1}<\cdots<b_{s}^{-1}$ denote the distinct roots of $Q$ for which some corresponding Ricci curvature $r_{i \bar{i}}$ is nonzero, where $a_{i}, b_{j}$ are positive. Let

$$
S(U)=U \prod_{i=1}^{q}\left(1+a_{i} U\right) \prod_{j=1}^{s}\left(1-b_{j} U\right), \quad P(U)=U Q(U) / S(U),
$$

and

$$
\Psi(U)=\left(\frac{d^{2}}{d U^{2}}(Q \varphi)(U)\right) / P(U) .
$$

Then $\Psi$ is a polynomial of degree $q+s+1$ and $\Psi(a)=-k_{a} S^{\prime}(a)$ for $a$ a root of $S(U) / U$, where $k_{a} \in \mathbf{R}^{+}$since $r$ is nonnegative. We can see that $S^{\prime}(a) \neq 0$ and $>0$ (or $<0)$ if and only if $S^{\prime}<0$ (or $>0$ ) for the root before $a$ and after $a$ (if they exist). And $S^{\prime}(0)>0$, so $S^{\prime}\left(-a_{q}^{-1}\right)<0, S^{\prime}\left(b_{1}^{-1}\right)<0$; that is, $\Psi\left(-a_{q}^{-1}\right)>0, \Psi\left(b_{1}^{-1}\right)>0$. Now there are $q-1$ (or $s-1$ ) zero points of $\Psi$ in $\left(-a_{1}^{-1},-a_{q}^{-1}\right)$ (or in $\left(b_{1}^{-1}, b_{s}^{-1}\right)$ ) if $q, s$ are not zero (one may also check the case of $s=0$ or $q=0)$. If $\varphi$ has nonpositive points in $(-d, D)$, then in $(-d, D) Q \varphi$ has at least two maximal points and one minimal point, since $\varphi(-d)=\varphi(D)=0, \varphi(-d+\varepsilon)>0, \varphi(D-\varepsilon)>0$ for $\varepsilon$ small enough. So we get that there are at least 4 zero points of $\Psi$ in $\left(-a_{q}^{-1}, b_{1}^{-1}\right) . \Psi$ has at least $(q-1)+(s-1)+4=q+s+2$ zero points, i.e., $\Psi(U)=0, Q \varphi$ is linear, so $Q \varphi=0$, a contradiction. Q.E.D.

By the same method as in [KS1], the theorem is proved.

Corollary 1. On $\widehat{L}$ there exists a Kähler metric with constant scalar curvature in the given class of $\tilde{g}$ if and only if the Futaki invariant is zero.

Theorem 2. For every Kähler class of a compact almost homogeneous manifold with two ends, there exists a unique extremal metric up to automorphisms.

Proof. Since every compact Kähler almost homogeneous space with two ends is a completion of a $C^{*}$ bundle over a product of a torus $A$ and a $C$-space $N$ with two homogeneous Kähler spaces as two ends [HS, Theorem 3.2], a maximal compact subgroup of the identity component of the automorphism group of this manifold is $G=A \times S \times S^{1}$, where $A$ is also the Albanese torus and $S$ is a maximal compact subgroup of the identity component of the automorphism group of $N$. For any Kähler metric $g, g_{G}=\int_{h \in G} h^{*} g d m$ is a Kähler metric of the form (1), where $m$ is the Haar measure on $G$; it is invariant under $G$. And also the Ricci curvature of $A \times N$ is nonnegative, so the condition in our assumption follows from the well-known property of the invariant cohomology $(1,1)$ classes for these manifolds (see [DG, p. 326, proof of the proposition], for example).

A result of Calabi [Cl2] says that we may assume that the extremal metric is invariant under $G$; that is, every extremal metric has the form (1). So the uniqueness follows from Lemma 5. Q.E.D.

Theorem 3. The completion of any multicanonical $C^{*}$ bundle over a KählerEinstein manifold of positive Ricci curvature admits an extremal metric.

Proof. By the main theorem and the argument in the proof of Theorem 5.4 in [KS1, p. 177]. 
Remark 1 (cf. [Mb]). In the above case we have

$$
s=s(U)=\int_{0}^{U} \kappa(U) d U, \quad t=t(U)=\int_{0}^{U} \kappa^{1 / 2}(U) d U,
$$

where $\kappa(U)=Q(U) / \Phi(U)$; so $U=s^{-1}(s)$.

Remark 2. In the general case we have

$$
F(H)=2\left[-D Q(D)+d Q(-d)-\delta_{1}+[Q(D)+Q(-d)+\delta] \beta / \alpha\right],
$$

where $F(H)$ is the Futaki invariant, $\delta=\int_{-d}^{D} \Delta(U) d U, \delta_{1}=\int_{-d}^{D} U \Delta(U) d U$. And in the case that $g_{0}$ is Fano $\Delta=n Q-U Q^{\prime}$, so $\delta=(n+1) \alpha-D Q(D)-$ $d Q(-d), \delta_{1}=(n+2) \beta-D^{2} Q(D)+d^{2} Q(-d)$.

Remark 3. In [Ki] N. Koiso also considered the extremal metrics in the Fano case, but he miscalculated the Laplacian. Here we want to say that a quasiEinstein metric is extremal if and only if it is Kähler-Einstein. First, a quasiEinstein metric must be in the first Chern class up to a constant, since every one-parameter group fixes all $Z$ cohomology classes. In the quasi-Einstein case Koiso's solution shows that $Q \varphi$ is a polynomial of degree $\operatorname{deg} Q+2$, but for the extremal metrics $Q \varphi$ has degree $\operatorname{deg} Q+3$ except $a=0$, i.e., the scalar curvature is constant, which must be Einstein in the Fano case. A generalization of Koiso's quasi-Einstein metric has been considered in [Gu 1, 2].

\section{REFERENCES}

[Bu] D. Burns and P. de Bartolomeis, Stability of vector bundles and extremal metrics, Invent. Math. 92 (1988), 403-407.

[Cl1] E. Calabi, Extremal Kähler metrics, Seminars on Differential Geometry, Ann. of Math. Stud., no. 102, Princeton University Press, Princeton, NJ, 1982, pp. 259-290.

[C12] _ Extremal Kähler metrics. II, Differential Geometry and Complex Analysis, SpringerVerlag, Berlin, 1985, pp. 95-114.

[DG] J. Dorfmeister and Z. Guan, Fine structure of reductive pseudo-Kählerian spaces, Geom. Dedicata 39 (1991), 321-338.

[FMS] A. Futaki, T. Mabuchi, and Y. Sakane, Kähler-Einstein metrics with positive Ricci curvature, Kähler Metric and Moduli Spaces, Adv. Stud. Pure Math., vol 18-II, Academic Press, Boston, MA, and Kinokuniya, Tokyo, 1990, pp. 11-83.

[Gu1] Z. Guan, On certain complex manifolds, Dissertation, Univ. of California, Berkeley, CA, 1993.

[Gu2] _-, Quasi-Einstein metrics, Internat. J. Math. (to appear).

[Gu3] _ Quasi-Einstein metrics and evolution of metrics in a Kähler class. I, in preparation.

[HS] A. Huckleberry and D. Snow, Almost-homogeneous Kähler manifolds with hypersurface orbits, Osaka J. Math. 19 (1982), 763-786.

[Hw] A. Hwang, Extremal Kähler metrics on almost-homogeneous spaces, preprint, 1992.

[Ki] N. Koiso, On rotationally symmetric Hamilton's equation for Kähler-Einstein metrics, Recent Topics in Differential and Analytic Geometry, Adv. Stud. Pure Math., vol. 18-I, Academic Press, Boston, MA, and Kinokuniya, Tokyo, 1990, 327-337.

[KS1] N. Koiso and Y. Sakane, Non-homogeneous Kähler-Einstein metrics on compact complex manifolds, Curvature and Topology of Riemannian Manifolds, Lecture Notes in Math., vol. 1201, Springer-Verlag, Berlin, 1986, pp. 165-179.

[KS2] _ Non-homogeneous Kähler-Einstein metrics on compact complex manifolds. II, Osaka J. Math. 25 (1988), 933-959. 
[Lv] M. Levine, A remark on extremal Kähler metrics, J. Differential Geom. 21 (1985), 73-77.

[Mb] T. Mabuchi, Einstein-Kähler forms, Futaki invariants and convex geometry on toric Fano varieties, Osaka J. Math. 24 (1987), 705-737.

[Sk] Y. Sakane, Examples of compact Kähler-Einstein manifolds with positive Ricci curvatures, Osaka J. Math. 23 (1986), 585-617.

Department of Mathematics, University of California, Berkeley, California 94720

Current address: Department of Mathematics, Princeton University, Princeton, New Jersey 08544

E-mail address: zguan@math.princeton.edu 